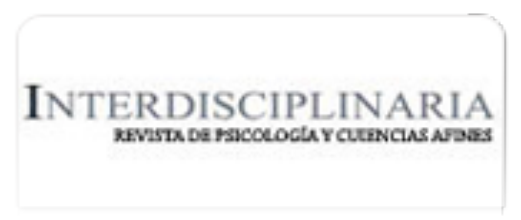

Interdisciplinaria

ISSN: 0325-8203

ISSN: 1668-7027

interdisciplinaria@fibercorp.com.ar

Centro Interamericano de Investigaciones Psicológicas y

Ciencias Afines

Argentina

Gariboldi, María Belén; Salsa, Analía M.

Conocimientos sobre los aspectos formales y referenciales del dibujo, la escritura y los numerales en la lectura compartida entre madres y niños pequeños

Interdisciplinaria, vol. 35, núm. 2, 2018, Julio-, pp. 477-494

Centro Interamericano de Investigaciones Psicológicas y Ciencias Afines

Argentina

Disponible en: https://www.redalyc.org/articulo.oa?id=18058785014

Cómo citar el artículo

Número completo

Más información del artículo

Página de la revista en redalyc.org

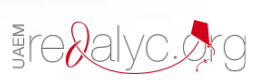

Sistema de Información Científica Redalyc Red de Revistas Científicas de América Latina y el Caribe, España y Portugal Proyecto académico sin fines de lucro, desarrollado bajo la iniciativa de acceso abierto 


\title{
Conocimientos sobre los aspectos formales y referenciales del dibujo, la escritura y los numerales en la lectura compartida entre madres y niños pequeños*
}

\section{Knowledge about the formal and referential aspects of drawing, writing and numerals during mothers and young children shared book reading}

\author{
María Belén Gariboldi** y Analía M. Salsa***
}

\begin{abstract}
*Este trabajo fue realizado con la financiación otorgada por la Agencia Nacional de Promoción Científica y Tecnológica de Argentina (PICT $2012 \mathrm{~N}^{\mathrm{o}}$ 1319).

Las autoras agradecen a las madres y a los niños que participaron en la investigación. **Psicóloga y Profesora en Psicología. Becaria Doctoral del Consejo Nacional de Investigaciones Científicas y Técnicas (CONICET) en el Instituto Rosario de Investigaciones en Ciencias de la Educación (IRICE).

E-mail: mariabelengariboldi@gmail.com

***Doctora en Psicología. Investigadora Independiente del Consejo Nacional de Investigaciones Científicas y Técnicas (CONICET) en el Instituto Rosario de Investigaciones en Ciencias de la Educación (IRICE).

E-mail: salsa@irice-conicet.gov.ar
\end{abstract}

Instituto Rosario de Investigaciones en Ciencias de la Educación (IRICE).

Consejo Nacional de Investigaciones Científicas y Técnicas (CONICET)

Universidad Nacional de Rosario (UNR)

\section{Resumen}

Los niños construyen conocimientos sobre los sistemas de representación externa en interacción con adultos que obran como mediadores de sus procesos cognitivos. Distintas investigaciones muestran que los niños reconocen el dibujo a partir de los 2 años y medio y pueden diferenciar entre dibujo, escritura y numerales alrededor de los 4 años. En este estudio se examinan los conocimientos sobre las propiedades formales y la función referencial de estos tres sistemas que niños de 2 años y medio y 4 años despliegan junto con sus madres durante la lectura compartida de un libro. Para ello se utilizó un libro que presenta en cada página dibujos de un animal, su nombre escrito y el numeral correspondiente a la cantidad de animales (1 a 9). Los análisis se centran en el foco de atención de las díadas, los aspectos del conocimiento notacional y las bases comunicativo-educativas de las interacciones, en función de la edad de los ni- ños. Los resultados indican que las díadas centraron su atención en la función referencial del dibujo, como representación de la identidad del referente a los 2 años y medio, y de la identidad y la cantidad a los 4 años. Aunque la atención en la escritura y los numerales fue menor, las madres de los niños más pequeños brindaron información sobre las propiedades formales de los numerales, y las madres y los niños de 4 años elaboraron este aspecto del conocimiento de ambos sistemas. Se discuten estos resultados en relación con el potencial de la lectura compartida para la apropiación temprana del conocimiento notacional.

Palabras clave: Interacción materno-infantil; Lectura compartida; Sistemas de representación externa; Función referencial; Propiedades formales 


\begin{abstract}
Children acquire knowledge about external representational systems in interaction with adults who operate as mediators of their cognitive processes. Several studies have shown that 2.5-year-old children are able to recognize drawings, and 4-year-old children can distinguish between drawing, writing and numerals. The present study focuses on a developmental analysis of the knowledge about the formal properties and the referential function of these three systems that 2.5- and 4-year-old children unfold with their mothers during shared reading sessions. The referential function is the representational relation between the system and the referent. Each system has a referential function that is the result of a social convention: figurative drawing depicts the identity and characteristics of the referent, writing is a graphic representation of oral language and numerals represent numerical information. The formal properties include the name of the representational systems and their graphic units, the form of the strokes, their spatial disposition and their compositional rules. Our specific goals were: (1) to establish which representational system is the main focus of attention; (2) to describe and analyze whether and how mothers and children elaborate knowledge about the formal properties and referential function of the systems; (3) to understand how notational knowledge emerges describing the educative-communicative basis of the interactions; and (4) to compare the focus of attention, notational knowledge and the educative-communicative basis of interactions as a function of children's age. Twenty-six mothers and their 2.5- $(n=13)$ and 4-year-old children ( $n$ $=13$ ) participated. They were given a book and told to look at it together. The book was specially designed for this study; it includes in each page drawings of an animal, its written name and the numeral for the number of animals depicted (1 to 9). We designed a system of categories with three levels of analysis, related to the specific goals of the study: focus of attention, notational knowledge and educative-communicative basis. We performed non-parametric statistical analysis: Wilcoxon test and Mann-
\end{abstract}

Whitney's $U$ test. Results show that dyads of both age groups focused their attention on drawings more than on writing and numerals. However, 4-year-old children and their mothers made significantly more utterances about writing and numerals than the other group. Attention to writing and numerals in the older group seemed to be guided by mothers' interest to teach those systems to their children. Dyads talked especially about the referential function of drawing, as a representation of the identity of the referent at 2.5 years of age and as a representation of the identity and quantity at 4 years. Although less attention was paid to writing and numerals, the youngest children's mothers provided information about the formal properties of numerals and the mothers and the 4-year-old children elaborated this aspect of knowledge of both representational systems. Furthermore, in the older group, dyads started to discuss the referential function of numerals. With regard to the educative-communicative basis of the interactions, the mothers of both age groups tended to request information about the referents of drawings more than to provide their children with this kind of information. The mothers in the 2.5year-olds' group provided information about the formal properties of numerals, while in the 4year-olds' group the mothers both requested and provided this kind of information. Finally, the formal properties of writing were elaborated only by mothers in the older group. We discuss these results in terms of the potential of shared reading for the early acquisition of notational knowledge.

Keywords: Mother-infant interaction; Shared reading; External representational systems; Referential function; Formal properties

\section{Introducción}

La alfabetización es un proceso clave para el desarrollo personal y la participación sociocultural, que en las sociedades actuales comienza con el nacimiento y se extiende más allá de la infancia (Bazerman, 2013; Márquez, Iparraguirre \& Bengtsson, 2015; Olson, 1999). Desde una perspectiva 
multimodal, este proceso involucra el aprendizaje, la enseñanza y el uso de una variedad amplia de lenguajes verbales y no verbales que se preservan gráficamente, entre ellos el dibujo, la escritura y la notación numérica (Kress, 2010; Teubal \& Guberman, 2014). En fases iniciales de este proceso, los niños necesitan aprender dos aspectos de estos sistemas de representación externa, su función referencial y sus propiedades formales (Tolchinsky, 2007). En este estudio el interés se centra en estos dos aspectos del conocimiento notacional en situaciones informales de aprendizaje. Si bien algunos enfoques teóricos diferencian los conceptos de representación externa y notación, se utilizará el de conocimiento notacional conservando el término empleado en Tolchinsky Landsmann y KarmiloffSmith (1992).

Entre las distintas funciones pragmáticas y epistémicas de los sistemas, la función referencial alude a la relación de representación entre las representaciones externas y sus referentes. Cada sistema posee una función referencial que es producto de una convención social. El dibujo figurativo denota la identidad y las características de objetos reconocibles e incluso nombrables. La escritura es una representación gráfica de los aspectos fonéticos, gramaticales, semánti$\cos$ y enunciativos del lenguaje oral y los numerales representan información numérica, con significados de etiquetación, cuantificación u orden. Las propiedades formales incluyen el nombre de los sistemas de representación y de sus unidades gráficas, las características de los trazos, su disposición espacial y reglas de composición que deben respetarse para que los sistemas cumplan con su función referencial. El dibujo ofrece una representación gráfica analógica, basada en criterios de semejanza culturalmente construidos, de aspectos de su referente como el contorno, partes y detalles cruciales para su identificación. Si bien se sostiene que el dibujo figurativo emplea un conjunto de trazos que se combinan según cierta sintaxis que requiere un aprendizaje, este conjunto tiene un carácter laxo y a- bierto. En contraste, la escritura y la notación numérica utilizan un conjunto limitado de unidades gráficas y reglas y principios de composición específicos. La escritura alfabética se basa en establecer correspondencias entre los fonemas del lenguaje oral y caracteres arbitrarios (los grafemas) y en combinar los grafemas en palabras y estas últimas en estructuras textuales de orden superior. Los numerales arábigos son un conjunto reducido de unidades gráficas arbitrarias (los dígitos del 1 al 9 y el 0) que se organizan según el principio posicional y la base 10 (Martí, 2003; Scheuer, de la Cruz \& Iparraguirre, 2010; Tolchinsky, 2007).

Desde un punto de vista evolutivo, Tolchinsky Landsmann y Karmiloff-Smith (1992) sostienen que los niños serían sensibles tempranamente a las propiedades formales de los sistemas, abordándolos como dominios de conocimiento antes de saber usarlos como instrumentos de comunicación según sus funciones referenciales convencionales. Ahora bien, los estudios que examinaron los conocimientos infantiles sobre las propiedades formales y la función referencial del dibujo, la escritura y los numerales han empleado tareas de naturaleza muy diversa. En tareas de selección y categorización, los niños demostraron basarse en las características formales para diferenciar, a partir de los 2 años y medio, el dibujo de la escritura y los numerales, y alrededor de los 4 años los tres sistemas entre sí (Ferreiro \& Teberosky, 1979; Tolchinsky Landsman \& Karmiloff-Smith, 1992; Yamagata, 2007).

En tareas de producción gráfica, los resultados de distintos estudios no son concluyentes. Brenneman, Massey, Machado y Gelman (1996) observaron en niños de 4 años una diferenciación entre dibujo y escritura basada en sus propiedades formales, particularmente en el tipo de trazos y su disposición espacial. Yamagata (2007) encontró esta producción diferenciada para dibujo, escritura y numerales en niños de 2 años; sin embargo, Levin y Bus (2003) mostraron que niños menores de 3 años producían marcas indistinguibles entre sí para dibujo y escritura. 
Otras investigaciones adoptaron una perspectiva diferente, solicitando a los niños que anotaran distintos tipos de información sin indicarles los sistemas que debían utilizar. Con la consigna de crear una tarjeta de identidad, niños de 3 años usaron dibujos, escritura y numerales según sus funciones referenciales para hacer un dibujo de sí mismos y comunicar información sobre el nombre, color de ojos, edad y número telefónico (Dockrell \& Teubal, 2007). No obstante, Martí y Garcia-Mila (2010) reportaron que niños de 4 años producían intercambios funcionales utilizando, por ejemplo, el dibujo para representar funciones de los numerales como la cantidad de objetos que contenía una caja.

En conjunto, estas investigaciones muestran, por un lado, una diferenciación de los sistemas que se adquiriría progresivamente entre los 2 y 4 años; por otro, que el contexto de uso de las representaciones que se ofrece a los niños restringiría los conocimientos que ponen en juego sobre las propiedades formales y la función referencial de los sistemas.

El propósito en este estudio fue describir y analizar los conocimientos notacionales sobre dibujo, escritura y numerales que niños de 2 años y medio y 4 años despliegan junto con sus madres en una actividad frecuente en la vida cotidiana de las díadas: la lectura compartida de un libro. Se partió de la idea de que los niños se acercan a las funciones y usos de los sistemas de representación antes de dominar las convenciones de su producción e interpretación en un proceso habilitado y promovido por la interacción con otros en contextos informales de aprendizaje (Martí, 2003; Teubal \& Guberman, 2014; Tomasello, 1999).

En las sociedades occidentales y niveles socioeconómicos medios, la lectura compartida es un formato de interacción entre padres e hijos presente ya antes del primer año de vida del niño. Esta actividad es utilizada por los adultos como una experiencia de enseñanza-aprendizaje en la cual guiar la atención y participación de los niños, promoviendo el desarrollo del lenguaje oral en particular y de la cognición en general (Fletcher \& Reese, 2005; Mareovich, Taverna \& Peralta, 2015; Ninio, 1983; Peralta \& Salsa, 2001). Al igual que en otras situaciones de interacción, los adultos guían a los niños durante la lectura compartida mediante estrategias comunicativo-educativas que persiguen diferentes intenciones (cognitivas, afectivas y metacognitivas) y se valen de diversos medios: dar feedback o retroalimentación al niño sobre su desempeño, brindar instrucciones explícitas sobre qué debe hacer y cómo, brindar pistas o sugerencias para ayudarlo a avanzar en la resolución de una tarea, modelar o demostrar ofreciendo un comportamiento a imitar y formular preguntas generando respuestas activas por parte del niño (Van de Pol, Volman \& Beishuizen, 2010).

Algunas investigaciones han usado la lectura compartida para explorar los conocimientos que padres y niños pequeños elaboran sobre el dibujo (Szechter \& Liben, 2004) y el número (Anderson, Anderson \& Shapiro, 2005). En la literatura, una atención mayor han recibido los conocimientos sobre escritura desplegados en este tipo de interacción y su relación con las habilidades de alfabetización emergente (Aram \& Levin, 2002; Deckner, Adamson \& Bakeman, 2006; Demir, Applebaum, Levine, Petty \& Goldin-Meadow, 2011; Whitehurst \& Lonigan, 1998). Los estudios que compararon dos tipos de representación se focalizan en dibujo y escritura, e indican que madres y niños tienden a centrar su atención en las ilustraciones, observándolas por más tiempo, describiéndolas o realizando más preguntas sobre ellas que sobre el texto escrito (Evans \& Saint-Aubin, 2005; Shapiro, Anderson \& Anderson, 1997; Yaden, Smolkin \& Conlon, 1989).

El presente estudio se diferencia de estas investigaciones en al menos dos aspectos. Por un lado, una aproximación novedosa en los estudios de interacción materno-infantil es examinar los conocimientos sobre dibujo, escritura y numerales en forma conjunta; por otro lado, se diferenciaron los conocimientos puestos en juego por madres 
y niños en función de si aludían a las propiedades formales de los sistemas o a su función referencial. Tal como se mencionó anteriormente, hasta el momento estos aspectos del conocimiento notacional han sido explorados en tareas de categorización y de producción que los niños resolvían en forma independiente.

Para ello, se diseñó un libro que posee en cada página un dibujo de una colección de animales, el nombre escrito del animal y el numeral correspondiente a la cantidad. Por lo tanto, la función referencial del dibujo es doble: representa la identidad del referente, permitiendo a las díadas elaborar información lingüística al nombrarlo, describirlo, clasificarlo o relacionarlo con experiencias previas, y representa la cantidad mediante la repetición de elementos idénticos. La escritura denota la identidad del referente a través de su nombre y los numerales representan el valor cardinal de las colecciones de animales con una única representación para referir a una pluralidad.

Los objetivos específicos del presente estudio fueron: (1) determinar qué sistema de representación constituye un foco de atención privilegiado para las díadas; (2) describir y analizar si las madres y sus niños ponen en juego las propiedades formales y la función referencial de los sistemas y cómo lo hacen; (3) comprender cómo emergen estos conocimientos describiendo las bases comunicativo-educativas de las interacciones, y (4) comparar el foco de atención, los conocimientos notacionales y las bases comunicativo-educativas en función de la edad de los niños.

\section{Método}

\section{Participantes}

En este estudio participaron 26 madres y sus niños de 2 años y medio $(n=13, M=$ 29.7 meses) y de 4 años $(n=13, M=48.5$ meses). La selección de estos grupos etarios se fundamenta en los hitos evolutivos que se mencionaron en la introducción: la discri- minación del dibujo alrededor de los 2 años y medio y la diferenciación entre los tres sistemas en torno a los 4 años. Se contactaron a las díadas a través de los establecimientos educativos a los que concurrían los niños, instituciones de la ciudad de Rosario (Argentina). Los niños no contaban con antecedentes de trastornos en su desarrollo cognitivo y/o del lenguaje, de acuerdo con lo informado por sus madres y los registros de las instituciones educativas.

La media de edad de las madres era de 32.65 años. Con respecto a sus niveles de escolaridad, 6 madres (23\%) poseían estudios secundarios completos, 4 (15\%) estudios terciarios y/o universitarios incompletos y 16 madres $(62 \%)$ estudios terciarios y/o universitarios completos. En cuanto a su ocupación, 10 madres (38\%) trabajaban en sus profesiones, 7 (27\%) en relación de dependencia, $4(16 \%)$ eran comerciantes y 5 madres (19\%) se desempeñaban como amas de casa.

\section{Materiales}

El libro posee nueve páginas de cartón $(20 \times 20 \mathrm{~cm})$ con dibujos de animales familiares para los niños en posición central, sus nombres en letra imprenta mayúscula y los numerales arábigos que denotan la cantidad de animales, ordenados de manera creciente del 1 al 9 (Figura 1). Las palabras y los numerales aparecen en diferentes posiciones en las páginas intercalando arriba y abajo de la ilustración. En cada página se utiliza el mismo color para los dibujos (sin rellenar las formas), los numerales y la escritura, variando los colores por página.

\section{Procedimiento}

Las sesiones de lectura se realizaron en una sala disponible del establecimiento educativo al que concurría el niño o en el hogar, según la preferencia de la madre; cada sesión tenía una duración aproximada de 15 minutos. El niño se sentaba junto a su madre y la experimentadora se ubicaba detrás y a la derecha de la díada para compartir 
con ellos la visión del libro y no interferir. Al entregarles el libro, la única consigna que se le daba a la pareja era que lo miraran juntos.

Respecto de los resguardos éticos, en primer lugar el personal directivo de los establecimientos educativos brindó su consentimiento para contactar a las madres, a quienes luego se les informó acerca de los requerimientos del estudio y se les solicitó autorizar en forma escrita su participación junto con sus hijos, asegurándoles confidencialidad de la información.

\section{Codificación y análisis de datos}

Se registraron las sesiones de lectura con una filmadora y luego fueron transcriptas textualmente para su posterior codificación y análisis. Se creó un sistema de codificación aplicando el método comparativo constante (Glaser \& Strauss, 1967). Este método permite identificar las propiedades específicas del fenómeno en estudio y generar inductivamente categorías abiertas y revisables a través del análisis iterativo de los datos en varias etapas. El sistema de codificación es producto de tres niveles de análisis de las sesiones de lectura, en línea con los objetivos del estudio.

\section{Foco de atención.}

En este primer nivel, en cada intervención de la madre y del niño se identificaba si aludía a Dibujo (D), Escritura (E) o Numerales $(\mathrm{N})$. Cuando la madre formulaba una pregunta abierta que no se centraba en un sistema en particular (por ejemplo, ‘¿Qué hay aquí?’ sin acompañar la verbalización con un gesto de señalar), se codificaron estas intervenciones de acuerdo al sistema referido por el niño en su respuesta. Las tres categorías construidas fueron exhaustivas y mutuamente excluyentes debido a que no se registraron intervenciones que establecieran relaciones explícitas entre los sistemas.

\section{Conocimiento notacional.}

En el segundo nivel de análisis se identificó qué aspecto del conocimiento sobre cada sistema se ponía en juego en las intervenciones de la madre y del niño: las Propiedades Formales o la Función Referencial. En la Tabla 1 se presentan las categorías resultantes de la confluencia de los niveles de análisis foco de atención y conocimiento notacional. Cabe señalar que se fusionó la conducta verbal con la no verbal debido a la frecuencia baja de gestos con información sobre los sistemas (por ejemplo, comunicar una cantidad empleando los dedos).

Las intervenciones referidas a las Propiedades Formales (categoría Sistema) consistían en nombrar el sistema, identificar sus unidades (letras y dígitos), describir los trazos o cómo se despliegan en el espacio.

El aspecto Función Referencial posee tres categorías:

- Identidad: intervenciones de la madre o del niño que elaboraban información sobre la identidad del referente (animales) a partir del dibujo o de la palabra escrita.

- Cantidad: intervenciones que elaboraban información sobre la cantidad (el valor cardinal de la colección de animales) a partir del dibujo o de los numerales.

- Relación: intervenciones que elaboraban información vinculando las representaciones o sus significados con experiencias previas de las díadas.

\section{Bases comunicativo-educativas.}

Se realizó un tercer nivel de análisis considerando en cada intervención materna el medio empleado para apoyar cognitiva y/o afectivamente la producción de los niños, tomando como marco la propuesta de Van de Pol, Volman y Beishuizen (2010). En base al análisis de las interacciones observadas, construimos las siguientes categorías:

- Brindar información. Afirmaciones de la madre que proporcionaban información al niño sobre los sistemas: 'Son leones', 'Este es el siete', 'Acá dice pollitos'. 
- Demandar información. La madre solicitaba información al niño en forma de pregunta ('¿Qué son esos?' señalando un dibujo, ‘¿Qué letra es?’, ¿Cuántos pollitos hay?') o con una instrucción explícita ('Cuéntalos').

- Corregir. La madre corregía una intervención del niño al considerarla errónea o inadecuada, de forma directa ('No, no es una $A$ ') o indirecta (' $¿$ Te parece que es el 9?').

- Evaluar positivamente. Tras una intervención correcta del niño, la madre la repetía indicando su aceptación ('Un perro') o la evaluaba positivamente ('Sí, así es', ‘¡Muy bien!').

Las intervenciones del niño fueron analizadas en relación con las intervenciones de su madre, registrando en cada categoría infantil si ésta era espontánea, una imitación de una intervención materna previa o si había sido requerida por la madre.

Se controló la fíabilidad del sistema de codificación seleccionando al azar un tercio de las sesiones para su codificación independiente por parte de dos investigadoras que no participaron en la construcción del sistema. El porcentaje de acuerdo alcanzado para el conjunto de las categorías fue superior al 95\%. En los casos de desacuerdo se alcanzó acuerdo luego de un chequeo conjunto.

Para los análisis estadísticos se emplearon pruebas no paramétricas debido al número de díadas y la distribución asimétrica de los datos: la prueba de Wilcoxon para muestras relacionadas y la prueba $U$ de Mann-Whitney para muestras independientes.

\section{Resultados}

Se organizó el reporte de los resultados en función de los objetivos específicos del estudio. En el primer apartado se presentan los resultados relativos al foco de atención de las díadas (objetivo 1) y en el segundo, los resultados acerca de cómo madres y niños pusieron en juego conocimientos sobre las propiedades formales y la función referencial de los sistemas (objetivo 2). En el tercer apartado se describen las bases comunicativo-educativas de las interacciones (objetivo 3). En cada uno de estos apartados, se integraron los análisis de comparación por grupo de edad (objetivo 4).

\section{Foco de atención}

Para determinar cuál fue el sistema foco de atención privilegiado para las díadas se consideró el número total de intervenciones de las madres y los niños en forma conjunta. En los dos grupos la atención estuvo centrada en el dibujo: estas intervenciones (Mdns 2;6 años: 66; 4 años: 70) fueron superiores a las de escritura (Mdns 2;6 años: 1; 4 años: $9 ; Z=-3.181, p=.001$ y $Z=-3.111$, $p=.002$, respectivamente) y numerales (Mdns 2;6 años: 3; 4 años: 9; $Z=-3.180, \mathrm{p}=$ .001 y $Z=-3.181, p=.001$, respectivamente). Además, también en ambos grupos, no hubo diferencias entre el número de intervenciones sobre escritura y numerales $(Z=-1.362$, $p=.173$ y $Z=-0.105, p=.916$, respectivamente).

Las comparaciones por edad arrojaron diferencias a favor del grupo de 4 años en escritura $(U=45.5, p=.041)$ y numerales $(U$ $=43.5, p=.035)$ y no en dibujo $(U=72, p=$ $.521)$. En conjunto, estos datos muestran que si bien el dibujo fue el foco de atención principal para las díadas, en el grupo de 4 años se incrementó el interés por la escritura y los numerales.

A partir de estos resultados se decidió analizar por separado las intervenciones maternas e infantiles para examinar la contribución de madres y niños en el interés creciente por la escritura y los numerales. Este análisis indica, por un lado, un aumento en el grupo de 4 años de las intervenciones maternas sobre estos sistemas (Mdns 2;6 años: Dibujo: 45, Escritura: 1, Numerales: 3; 4 años: Dibujo: 48, Escritura: 6, Numerales: 6) $(U=83, p=.939 ; U=44, p=.034$ y $U=41.5, p=.026)$. Por otro lado, consideramos las intervenciones espontáneas de los niños y no encontramos diferencias por 
edad (Mdns 2;6 años: Dibujo: 8, Escritura: 0, Numerales: 0; 4 años: Dibujo: 9, Escritura: 0, Numerales: 1$)(U=84, p=.98 ; U=$ $70, p=.31$ y $U=53.5, p=.07)$. En consecuencia, la atención hacia la escritura y los numerales en el grupo de 4 años parecería haber estado guiada por el interés de las madres en el aprendizaje de estos sistemas por parte de sus hijos.

\section{Conocimiento notacional}

En primer lugar se analizó qué aspectos del conocimiento se elaboraron durante las sesiones de lectura, considerando nuevamente las intervenciones maternas e infantiles en forma conjunta para conocer si la atención de las díadas se centró en las propiedades formales o en la función referencial de cada sistema (ver Tabla 2). Ante los dibujos, ambos grupos elaboraron únicamente información sobre su función referencial, sin aludir a sus propiedades formales identificándolo con su nombre o describiendo sus trazos y disposición espacial.

Para escritura y numerales encontramos un patrón de resultados opuesto. Los dos grupos se centraron en sus propiedades formales más que en su función referencial. Los análisis al interior de cada grupo evidenciaron diferencias entre propiedades formales y función referencial solamente a los 2 años y medio para escritura $(Z=-2.207, p=$ $.027)$ y numerales $(Z=-2.386, p=.017)$. Los análisis en función de la edad revelaron un incremento significativo a los 4 años de las intervenciones relativas a la función referencial de los numerales $(U=49.5, p=.05)$.

En segundo lugar, con respecto a los tipos específicos de información sobre los sistemas, se analizaron por separado las intervenciones maternas e infantiles para describir con mayor detalle las contribuciones de ambos integrantes de las díadas (Tabla $3)$. La categoría Sistema reunió las intervenciones sobre las propiedades formales. Las madres del grupo de 2 años y medio elaboraron información únicamente sobre las propiedades formales de los numerales, mientras que las madres del grupo de 4 años lo hicieron tanto para este sistema, como para la escritura. Observamos un patrón similar en los niños de 4 años, a diferencia de los niños de 2 años y medio, con la aparición de intervenciones sobre las propiedades formales de la escritura y los numerales. Las comparaciones por edad no resultaron significativas en las intervenciones maternas (ES: $U=49.5, p=.061$; NS: $U=60.5, p=$ .210) ni en las infantiles (ES: $U=58, p=$ .145 ; NS: $U=56.5, p=.138$ ).

En cuanto a la función referencial de los sistemas, la Tabla 3 muestra que las madres del grupo de 2 años y medio utilizaron el dibujo para referirse a la identidad de los animales en mayor medida que a la cantidad $(Z=-3.113, p=.002)$, tendencia que estaría cambiando en el grupo de 4 años al disminuir las referencias maternas a la identidad y aumentar las de la cantidad con soporte en el dibujo $(Z=-1.960, p=.059)$. En las intervenciones infantiles este patrón de resultados es más claro, con diferencias entre identidad y cantidad a los 2 años y medio ( $Z=$ $-3.183, p=.001)$ que desaparecen a $\operatorname{los} 4$ años $(Z=-0.175, p=.861)$.

Aunque la palabra escrita también denotaba la identidad de los animales, ni las madres ni los niños elaboraron esta información en ninguno de los grupos. Para los numerales y la cantidad, solamente se encontraron indicios de elaboración de esta relación en las madres del grupo de 4 años y con una frecuencia muy baja. En síntesis, ante la presencia de los dibujos, las díadas privilegiaron este sistema sobre la escritura y los numerales para elaborar información sobre la identidad de los referentes y su cantidad.

En línea con estos datos, las comparaciones por edad mostraron que las intervenciones maternas sobre la identidad a partir del dibujo disminuyeron en el grupo de 4 años con respecto al de 2 años y medio $(U=42, p=.029)$, mientras que aumentaron las que referían a la cantidad usando como soporte el dibujo $(U=37.5, p=.016)$ y los numerales $(U=51.5, p=.05)$. Asimismo, las intervenciones de los niños de 4 años sobre la información cuantitativa de los dibu- 
jos superaron a las de los niños de 2 años y medio $(U=16, p<.001)$. Sería posible pensar, entonces, que en el grupo de 4 años, madres y niños emplearon la sesión de lectura para elaborar información numérica, junto con información lingüística.

Finalmente, la categoría Relación apareció solo con el dibujo y como estrategia empleada por las madres $(U=57, p=.151)$ y los niños más pequeños $(U=51.5, p=$ .352). Un análisis más exhaustivo de estas intervenciones revela que las madres del grupo de 2 años y medio se refirieron a la identidad de los animales, particularmente a sus características, relacionando los dibujos con experiencias de sus hijos, como dibujos animados con el animal como protagonista, canciones en las que surge su nombre, mascotas y visitas al zoológico.

\section{Bases comunicativo-educativas}

Las intervenciones maternas fueron examinadas de acuerdo con el medio que empleaban para apoyar las producciones de los niños: brindar o demandar información, corregir y evaluar positivamente (Tabla 4). En cuanto al dibujo y su función referencial, las madres de los dos grupos tendieron a demandar esta información a sus hijos en vez de brindarla explícitamente, tanto en relación con la identidad de los animales como con la cantidad. Las comparaciones al interior de los grupos entre brindar y demandar información fueron significativas a los 2 años y medio para identidad $(Z=-2.694, p=$ $.007)$ y a los 4 años para identidad $(Z=-3.122$, $p=.002)$ y cantidad $(Z=-2.609, p=.009)$. Con este sistema, la mediana para brindar información fue superior a demandar información únicamente en Dibujo Relación a los 2 años y medio, aunque la diferencia entre ambas no fue significativa $(Z=-1.259, p=$ .208).

Para las propiedades formales de los numerales, los estadísticos descriptivos muestran que los apoyos maternos en el grupo de 2 años y medio consistieron en brindar información, mientras que en el grupo de 4 años las madres emplearon las dos estrate- gias, brindar y demandar $(Z=-.66, p=.509)$. Para escritura, únicamente se registraron intervenciones maternas demandando información sobre sus aspectos formales a los 4 años $(Z=-1.78, p=.075)$. En ambos casos, los apoyos maternos buscaron identificar y nombrar las unidades de los sistemas (dígitos y letras).

Las correcciones tras una intervención errónea del niño fueron poco frecuentes y solamente para dibujo. Por el contrario, las madres de ambos grupos desplegaron ayudas afectivas relacionadas con evaluaciones positivas de las intervenciones de sus hijos, fundamentalmente cuando éstas aludían a la función referencial del dibujo y las propiedades formales de los numerales. Las comparaciones por edad revelan un aumento en el grupo de 4 años de las estrategias maternas de demanda de información $(U=46, p=.047)$, corrección $(U=45, p=$ .034) y evaluación positiva ( $U=30, p=$ .004) en Dibujo Cantidad, y de demanda de información en Numerales Sistema ( $U=$ $49.5, p=.05)$.

Con respecto a las intervenciones infantiles, en ambos grupos éstas fueron requeridas por las madres en mayor medida que espontáneas, para la identidad de los dibujos como para la cantidad. Únicamente los niños de 2 años y medio realizaron algunas imitaciones. Para escritura y numerales, se registraron intervenciones infantiles a los 4 años y siempre requeridas por las madres. Por último, las comparaciones por edad señalan un aumento a los 4 años de las intervenciones espontáneas $(U=20, p=.001)$ y requeridas $(U=26, p=.003)$ en Dibujo Cantidad y una disminución de intervenciones espontáneas en Dibujo Identidad ( $U=37.5$, $p=.015)$.

\section{Discusión}

Los resultados muestran que la lectura compartida sería un contexto propicio para promover tempranamente el despliegue de conocimientos sobre el dibujo, la escritura y los numerales. De este modo, este estudio 
contribuye a las investigaciones evolutivoeducativas que enfatizan el papel del adulto en situaciones informales que favorecen la alfabetización al acercar a los niños a los usos de distintos lenguajes, verbales y no verbales (Kress, 2010; Martí, 2003; Teubal \& Guberman, 2014). A continuación, se describen los resultados obtenidos en cada sistema de representación.

En relación con el dibujo, madres y niños focalizaron principalmente su atención en este sistema, en línea con los resultados de estudios previos sobre lectura compartida con libros con ilustraciones y texto escrito (Evans \& Saint-Aubin, 2005; Shapiro et al., 1997; Yaden et al., 1989). El aspecto privilegiado del conocimiento fue su función referencial: las díadas usaron el dibujo como herramienta de comunicación, conversando sobre los animales representados. Las madres de ambos grupos de edad demandaron a sus niños información sobre la identidad de los animales (el nombre y las características del referente) y establecieron relaciones entre los dibujos y experiencias del niño; por lo tanto, el dibujo habría fomentado el uso de estrategias maternas para promover el lenguaje oral, particularmente el vocabulario. En el grupo de 4 años el dibujo sirvió, además, para que las madres demandaran información sobre la cantidad, estimulando la repetición de elementos idénticos el incentivo al conteo ('Cuéntalos') y la cardinalización de las colecciones ('¿Cuántos hay?’).

Esta tendencia en los resultados permite esbozar algunas conclusiones. Primero, el interés en la función referencial del dibujo podría relacionarse con las cualidades específicas de esta representación. A diferencia de los sistemas compuestos por unidades arbitrarias, como la escritura y los numerales, el dibujo figurativo ofrece una correspondencia basada en relaciones de semejanza con su referente. Esta "manifestación" del referente (Zechetto, 2003) parecería haber propiciado elaboraciones relativas a sus nombres y características. Si bien para algunos autores el dibujo se organiza como un sistema siguiendo ciertas reglas (Martí, 2003;
Scheuer, de la Cruz \& Iparraguirre, 2010), éstas son más abiertas y de interpretación más global que las reglas de composición de la escritura y los numerales, pudiendo explicarse así la ausencia de intervenciones sobre sus propiedades formales. Asimismo, podrían haber intervenido también factores socioculturales, si se considera que la enseñanza de los elementos y reglas de composición del dibujo figurativo no suele plantearse en el sistema educativo como un objetivo con la misma relevancia y exigencia que se da a la instrucción de las convenciones de la escritura y los numerales (Jolley, 2010).

Segundo, a pesar de que la correspondencia entre un sistema y el contenido representado no es fija ni única, al elaborar información cuantitativa en base al dibujo, se produjeron entre las díadas, intercambios funcionales entre sistemas, similares a los descriptos por Martí y Garcia-Mila (2010). De esta forma, como se ha observado en el desarrollo individual, también en las interacciones entre madres y niños preescolares la especificidad funcional de los sistemas de representación surgiría lenta y progresivamente. Además, estos datos estarían en sintonía con los momentos iniciales de la adquisición de las notaciones numéricas, cuando los niños representan una cantidad repitiendo marcas idénticas para más tarde abandonar esta correspondencia término a término y emplear una única representación arbitraria (Bialystok \& Cood, 1996; Sinclair, 1991).

En cuanto a los numerales y la escritura, el interés de las díadas se centró en sus propiedades formales: las madres del grupo de 2 años y medio brindaron información sobre los nombres de los numerales, mientras que las madres y los niños de 4 años desplegaron esta información junto con los nombres de las letras. Las intervenciones infantiles fueron siempre requeridas por las madres, quienes parecieron ajustar sus estrategias a las capacidades infantiles en tanto que alrededor de los 4 años los niños diferenciarían escritura de numerales (Ferreiro \& Teberosky, 1979; Tolchinsky, Landsman \& Karmiloff-Smith, 1992). Además, en este grupo de 4 años, las 
madres comenzaron a demostrar cierto interés por la función referencial de los numerales, estableciendo correspondencias entre las marcas gráficas y las cantidades representadas, aunque sin hacer alusión a la función referencial de la palabra escrita. Por otro lado, las madres tendieron a enseñar a sus hijos los numerales antes que la escritura; este patrón de resultados requiere sin duda un análisis más exhaustivo en investigaciones futuras que aborden el curso natural de las interacciones entre madres y niños pequeños con estos sistemas, en otros tipos de tareas y apelando a diseños longitudinales.

Un dato a destacar en relación con los conocimientos desplegados sobre la escritura y los numerales fue la variabilidad al interior de cada grupo de edad, reflejada en los valores mínimos y máximos reportados para estas categorías (ver Tabla 3). En la mitad de las díadas del grupo de niños más pequeños no se registraron intervenciones maternas y/o infantiles en las categorías para las propiedades formales de estos sistemas (Escritura Sistema y Numerales Sistema); no obstante, en la otra mitad se observaron entre 1 y 9 intervenciones en estas categorías. En el grupo de 4 años sucedió algo similar para las propiedades formales de la escritura (Escritura Sistema) y la función referencial de la escritura (Escritura Identidad) y los numerales (Numerales Cantidad), con elaboraciones solo en algunas de las díadas.

Esta variabilidad intragrupo en el despliegue de conocimientos sobre la escritura y los numerales, podría relacionarse con diversos factores, tales como el estilo de interacción característico de cada díada, las creencias y expectativas de las madres sobre los conocimientos notacionales de sus niños o la interpretación de la demanda de la tarea. En futuros estudios sería interesante abordar los factores mencionados y explorar su posible relación con los conocimientos desplegados por las díadas.

Más allá de estas diferencias al interior de cada grupo, la hipótesis de Tolchinsky Landsmann y Karmiloff-Smith (1992) sobre que los sistemas de representación son ela- borados primero por los niños como dominios de conocimiento y luego como herramientas referenciales, se articularía con lo observado para la escritura y los numerales. Las madres parecerían considerar que sus hijos necesitan acercarse a estos sistemas como objetos en sí, para después aprender que sirven para la expresión y comunicación. Cabría preguntarse si esta secuencia temporal estaría guiada por la lógica misma de adquisición de los sistemas de representación, como sostienen estas autoras, o por la influencia en las estrategias maternas de tradiciones sociales y escolares que priorizan la enseñanza de la escritura y los numerales desde un enfoque instrumental (Alsina \& Llach, 2012). Este tipo de enfoque reduce el aprendizaje de estos sistemas al dominio mecánico de las reglas de producción e interpretación de los códigos alfabético y numérico, sin considerar la importancia de los contextos de uso en los que escritura y número cobran significado. La enseñanza de estas representaciones necesitaría centrarse desde los momentos iniciales en sus potencialidades comunicativas y epistémicas, además de en sus propiedades formales (Scheuer, de la Cruz \& Iparraguirre, 2010; Teubal \& Guberman, 2014).

Como se mencionó con anterioridad, la elaboración de información lingüística durante la lectura compartida de libros ha sido documentada ampliamente (Fletcher \& Reese, 2005; Mareovich, Taverna \& Peralta, 2015; Ninio, 1983; Peralta \& Salsa, 2001). El presente estudio se alinea a dichas investigaciones al registrar interacciones centradas en forma predominante en los nombres y características de los animales representados. Sin embargo, resultaron de especial interés los conocimientos sobre la cantidad desplegados por las díadas, principalmente a partir del dibujo $\mathrm{y}$, en forma incipiente, a partir de los numerales. Estos conocimientos fueron un foco de atención importante en el grupo de 4 años, en el que todas las madres y todos los niños se refirieron a la cantidad al menos una vez en la sesión. En la literatura se ha descripto la 
forma en que la lectura de libros podría favorecer la adquisición de conceptos matemáticos en el ámbito escolar (Hong 1996; Young-Loveridge, 2004). Por lo tanto, sería interesante profundizar el estudio de la lectura compartida como un contexto de aprendizaje informal de conocimientos numéricos.

Para finalizar, cabe destacar algunas limitaciones de este estudio, particularmente la conformación de grupos relativamente reducidos de díadas y la homogeneidad en el nivel educativo de las madres que participaron. Por este motivo, un objetivo futuro es analizar situaciones de lectura compar- tida en díadas de diferentes entornos socioeducativos ya que distintas investigaciones señalan diferencias en el modo en que los padres enseñan a sus hijos letras (Treiman et al., 2015) y números (Gunderson \& Levine, 2011) en función de su nivel educativo. Estas aproximaciones permitirán obtener información valiosa sobre las interacciones educativas en la familia que estimulan la apropiación temprana de los sistemas de representación externa y construir puentes hacia la educación infantil, con el fin de potenciar formas de alfabetización inclusivas y multimodales, acordes a los desafíos sociales y comunicativos actuales.
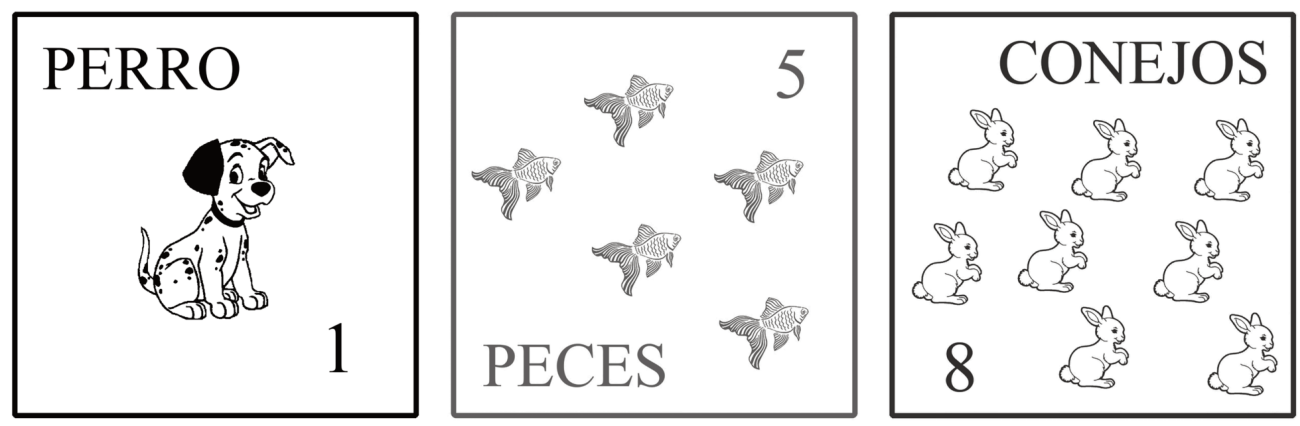

Figura 1. Ejemplos de páginas del libro diseñado para la investigación 
Tabla 1

Sistema de codificación y ejemplos de las categorías maternas e infantiles para Foco de Atención y Conocimiento Notacional

\begin{tabular}{|c|c|c|c|c|}
\hline Fure da & Cusaimien: & notucional & Cudips & Esmplos \\
\hline \multirow{4}{*}{ Ditujo } & $\begin{array}{l}\text { Fropicidaks } \\
\text { Fomules }\end{array}$ & Sistem & DS & "Eso es un dibup" \\
\hline & \multirow{3}{*}{$\begin{array}{l}\text { Funcikn } \\
\text { reflatmstal }\end{array}$} & Identited & Di & "Rorio", "Las pllins son amarilhs" \\
\hline & & Cratidad & DE & "Cuinkm polliwe hay", "Len, dos, tors" \\
\hline & & Rabion & DR & $\begin{array}{l}\text { Sal chunchise come las del dibjiin cque } \\
\text { nemos" }\end{array}$ \\
\hline \multirow{3}{*}{ Emonou } & $\begin{array}{l}\text { Fopicibia } \\
\text { homals }\end{array}$ & Sindemu & Es & 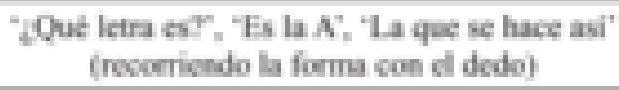 \\
\hline & \multirow{2}{*}{$\begin{array}{l}\text { Furkika } \\
\text { ecfercmelal }\end{array}$} & Idenlidad & EI & "Wue dice ahr? Diec pollike" \\
\hline & & Rabrion & EE & "La hater de Cori, comm la tia Cori" \\
\hline \multirow{3}{*}{ Numerales } & $\begin{array}{l}\text { Fropidudes } \\
\text { Cormales }\end{array}$ & Sistatu & MS & "Es un fümero" "Es el usa" \\
\hline & \multirow{2}{*}{$\begin{array}{l}\text { Funibin } \\
\text { etfercmetil }\end{array}$} & Cuntidud & We & 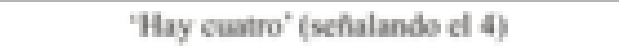 \\
\hline & & Relucion & NH & "Dom comw los ahos que tú tienes" \\
\hline
\end{tabular}

Tabla 2

Medianas, valores mínimos y máximos de intervenciones de las díadas sobre cada aspecto del Conocimiento Notacional, por grupo de edad

\begin{tabular}{|c|c|c|c|c|c|}
\hline \multirow{2}{*}{$\begin{array}{l}\text { Apwha del consinimio } \\
\text { mothionul }\end{array}$} & \multirow{2}{*}{ Bisetr } & \multicolumn{2}{|c|}{2, th ahm } & \multicolumn{2}{|c|}{4 ah้ก } \\
\hline & & Lut & Lism Mhr & Miln & Minth \\
\hline \multirow{3}{*}{ Propiodides for alos } & Dibujo & 0 & $\mathbf{0}=\mathbf{0}$ & 0 & $0=1$ \\
\hline & Fucribura & I & $0-12$ & 6 & $0-2 b$ \\
\hline & Numbrales & 3 & $0-3 k$ & 7 & $0-24$ \\
\hline \multirow{3}{*}{ Funcion reffmencial } & Dibujo & 62 & $40-119$ & 68 & $37-146$ \\
\hline & Escrour & 0 & $0=4$ & 0 & $0=35$ \\
\hline & Numanliss & 0 & $0-3$ & 2 & $0-20$ \\
\hline
\end{tabular}




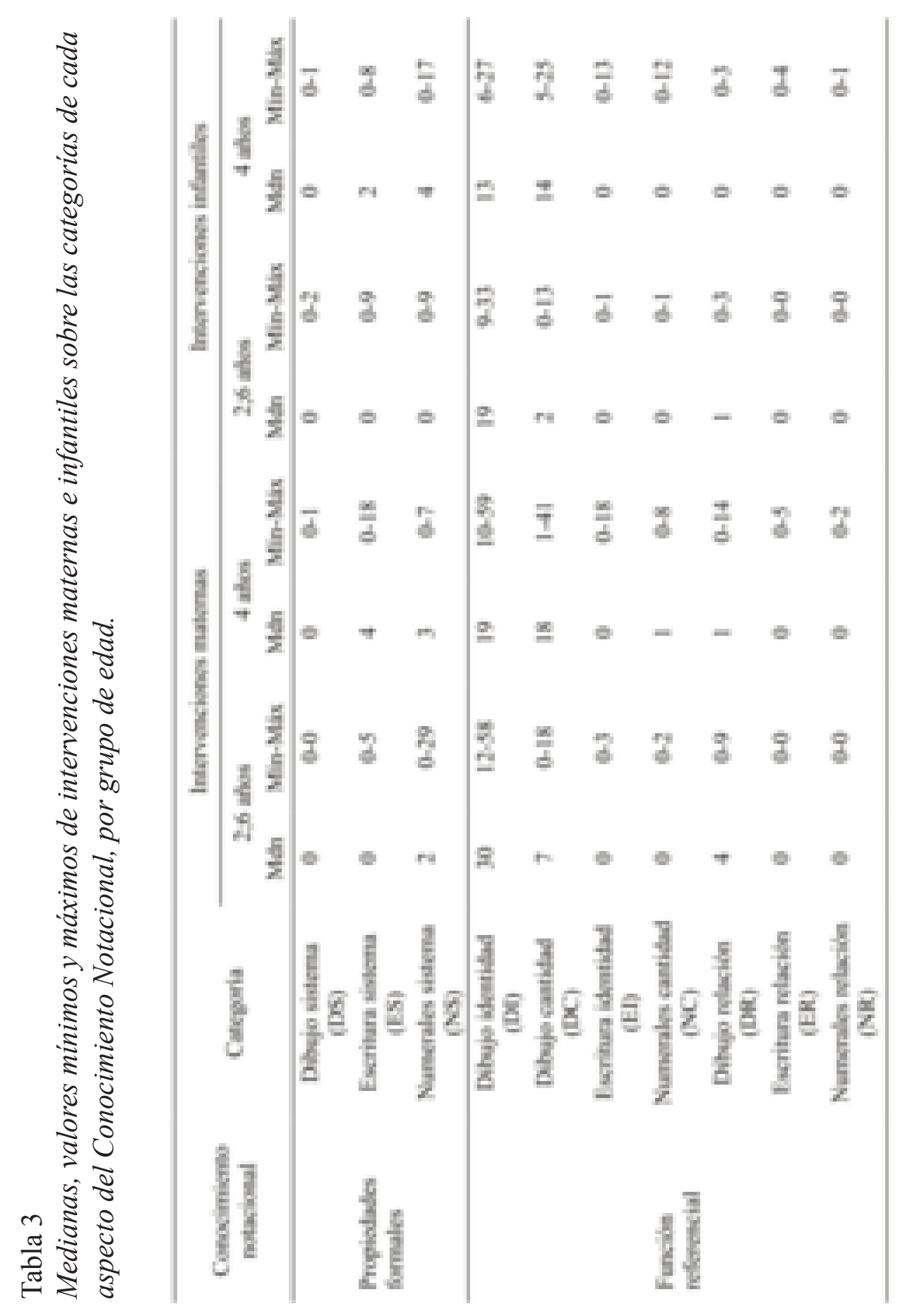




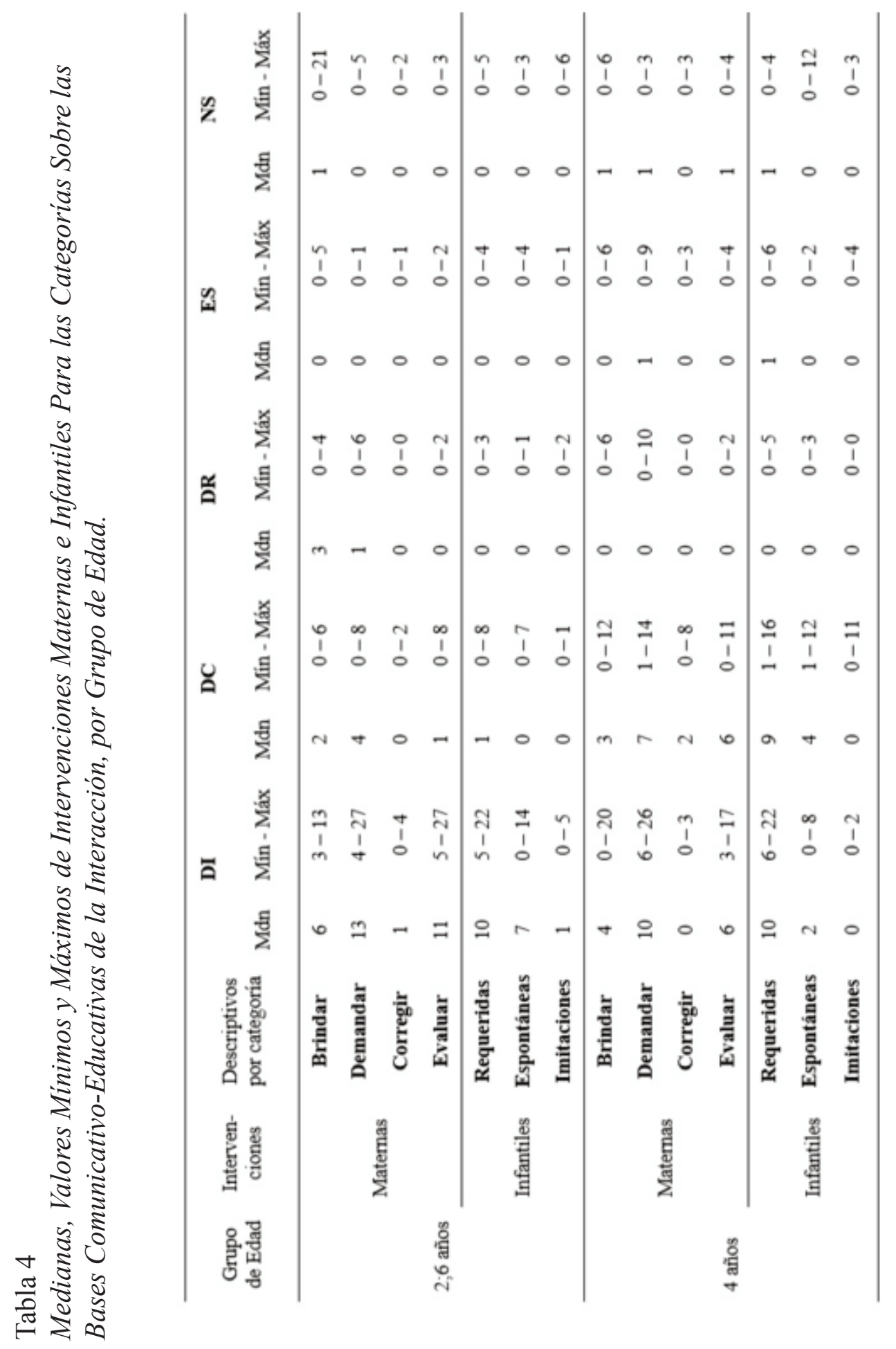




\section{Referencias bibliográficas}

Alsina, A. \& Llach, S. (2012). La enseñanza de los sistemas externos de representación matemáticos y lingüísticos en la educación infantil. Revista de Investigación Educativa, 30(1), 131-144. https://doi.org/10.6018/rie.30.1. 115121

Anderson, A., Anderson, J., \& Shapiro, J. (2005). Supporting multiple literacies: Parents' and children's mathematical talk within storybook reading. Mathematics Education Research Journal, 16, 5-26. https:// doi.org/10.1007/BF03217399

Aram, D., \& Levin, I. (2002). Mother-child joint writing and storybook reading: Relations with literacy among low SES kindergartners. Merrill Palmer Quarterly, 48, 202224. https://doi.org/10.1353/mpq.2002.0005

Bazerman, C. (2013). Understanding the lifelong journey of writing development. Infancia y Aprendizaje, 36, 421-441. https:// doi.org/10.1174/021037013808200320

Bialystok, E., \& Cood, J. (1996). Developing representations of quantity. Canadian Journal of Behavioural Science, 28, 281-291. https://doi.org/10.1037/0008-400X.28. 4.281

Brenneman, K., Massey, C., Machado, S., \& Gelman, R. (1996). Young children's plans differ for writing and drawing. Cognitive Development, 11, 397-419. https://doi.org/ 10.1016/S0885-2014(96)90011-8

Deckner, D., Adamson, L., \& Bakeman, R. (2006). Child and maternal contributions to shared reading: Effects on language and literacy development. Applied Developmental Psychology, 27, 31-41. https://doi.org/10. 1016/j.appdev.2005.12.001

Demir, O., Applebaum, L., Levine, S., Petty, K., \& Goldin-Meadow, S. (2011). The story behind parent-child book-reading interactions: Relations to later language and reading outcomes. En N. Danis, K. Mesh, \& H. Sung (Eds.), Proceedings of the 35th Boston
University Conference on Language Development (pp. 157-169). Somerville, MA: Cas cadilla.

Dockrell, J., \& Teubal, E. (2007). Distinguishing numeracy from literacy: Evidence from children's early notations. En E. Teubal, J. Dockrell, \& L. Tolchinsky (Eds.), Notational knowledge. Developmental and historical perspectives (pp. 113-134). Rotterdam: Sense.

Evans, M., \& Saint-Aubin, J. (2005). What children are looking at during shared storybook reading? Psychological Science, 16, 913-920. https://doi.org/10.1111/j.14679280.20 05.01636.x

Ferreiro, E., \& Teberosky, A. (1979). Los sistemas de escritura en el desarrollo del niño. Madrid: Siglo XXI.

Fletcher, K., \& Reese, E. (2005). Picture book reading with young children: A conceptual framework. Developmental Review, 25, 64103. https://doi.org/10.1016/j.dr.2004.08. 009

Glaser, B., \& Strauss, A. (1967). The discovery of grounded theory: strategies for qualitative research. Nueva York, USA: Aldine.

Gunderson, E., \& Levine, S. (2011). Some types of parent number talk count more than others: relations between parents' input and children's cardinal-number knowledge. Developmental Science, 14, 1021-1032. https:// doi.org/10.1111/j.1467-7687.2011.0 1050.x

Hong, H. (1996). Effects of mathematics learning through children's literature on math achievement and dispositional outcomes. Early Childhood Research Quarterly, 11, 477-494. https://doi.org/10.1016/S0885-20 06(96)90018-6

Jolley, R.P. (2010). Children and pictures: Drawing and understanding. West Sussex: Wiley-Blackwell.

Kress, G. (2010). Multimodality. A social-semiotic approach to contemporary communication. Oxon: Routledge.

Levin, I., \& Bus, A. (2003). How is emergent writing based on drawing? Analyses of chil- 
dren's products and their sorting by children and mothers. Developmental Psychology, 39, 891-905. https://doi.org/10.1037/ 00121649.39.5.891

Mareovich, F., Taverna, A. \& Peralta, O. (2015) Enseñando palabras mediante libros ilustrados: El aprendizaje temprano de sustantivos y adjetivos. Interdisciplinaria, 32(1), 151168.

Martí, E. (2003). Representar el mundo externamente. La adquisición infantil de los sistemas externos de representación. Madrid: Antonio Machado.

Martí, E., \& Garcia-Mila, M. (2010). Progresos en la diferenciación funcional entre dibujo, escritura y numerales en niños de 4 a 7 años. Estudios de Psicología, 31, 339-352. https://doi.org/10.1174/021093910793154420

Márquez, M.S., Iparraguirre, M.S. \& Bengt sson, A.M. (2015). Un recorrido por el aprendizaje de la escritura desde una perspectiva evolutivo-educativa y semiótica. Interdisciplinaria, 32(1), 151-168.

Ninio, A. (1983). Joint book reading as a multiple vocabulary acquisition device. Developmental Psychology, 19, 445-451. https:// doi.org/10.1037/0012-1649.19.3.445

Olson, D.R. (1999). El mundo sobre el papel. El impacto de la escritura y la lectura en la estructura del conocimiento. Barcelona: Gedisa.

Peralta, O., \& Salsa, A. (2001). Interacción materno-infantil con libros con imágenes en dos niveles socioeconómicos. Infancia $y$ Aprendizaje, 24, 325-339. https://doi.org/ 10.1174/021037001316949257

Scheuer, N., de la Cruz, M. de la \& Iparraguirre, M. (2010). El aprendizaje de distintos dominios rotacionales según niños de preescolar y primer grado. Revista Latinoamericana de Ciencias Sociales, Niñez y Juventud, 8(2), 1083-1097.

Shapiro, J., Anderson, J., \& Anderson, A. (1997). Diversity in parental storybook rea- ding. Early Child Development and Care, 127, 47-58. https://doi.org/10.1080/030044 3971270105

Sinclair, A. (1991). Children's production and comprehension of written numerical representations. En K. Durkin, \& B. Shire (Eds.), Language in mathematical education (pp. 5968). Buckingham: Open University Press.

Szechter, L., \& Liben, L. (2004). Parental guidance in preschoolers' understanding of spatial-graphic representations. Child Development, 75, 869-885. https://doi.org/10. 1111/j.1467-8624.2004.00711.x

Teubal, E., \& Guberman, A. (2014). Textos gráficos y alfabetización múltiple. Herramientas para el desarrollo del pensamiento y el aprendizaje en el nivel inicial. Barcelona: Paidós.

Tolchinsky, L. (2007). The multiple functions of external representations: Introduction. En E. Teubal, J. Dockrell, \& L. Tolchinsky (Eds.), Notational knowledge. Developmental and historical perspectives (pp. 1-10). Rotterdam: Sense.

Tolchinsky Landsmann, L., \& Karmiloff-Smith, A. (1992). Children's understanding of notations as domains of knowledge versus referential-communicative tools. Cognitive Development, 7, 287-300. https://doi.org/ 10.1016/0885-2014(92)90017-L

Tomasello, M. (1999). The cultural origins of human cognition. Cambridge, MA: Harvard University Press.

Treiman, R., Schmidt, J., Decker, K., Robins, S., Levine, S., \& Demir, Ö (2015). Parents' talk about letters with their young children. Child Development, 86, 1406-1418. https:// doi.org/10.1111/cdev.12385

Van de Pol, J., Volman, M. \& Beishuizen, J. (2010). Scaffolding in Teacher-Student Interaction: A Decade of Research. Educational Psychology Review, 22(3), 271-296. https://doi.org/10.1007/s10648-010-9127-6

Whitehurst, G.J., \& Lonigan, C.J. (1998). Child development and emergent literacy. Child 
Development, 69, 848-872. https://doi.org/ 10.1111/j.1467-8624.1998. tb06247.x

Yaden, D., Smolkin, L., \& Conlon, A. (1989). Preschoolers' questions about pictures, print conventions and story texts during reading aloud at home. Reading Research Quarterly, 24, 189-214. https://doi.org/10.2307/747864 Yamagata, K. (2007). Differential emergence of representational systems: Drawings, letters, and numerals. Cognitive Development, 22,
244-257. https://doi.org/10.1016/j.cogdev. 2006.10.006

Young-Loveridge, J. (2004). Effects on early numeracy of a program using number books and games. Early Childhood Research Quarterly, 19, 82-98. https://doi.org/10.10 16/j.ecresq.2004.01.001

Zecchetto, V. (2003). La danza de los signos. Nociones de semiótica general. Buenos Aires: La Crujía.

Recibido: 6 de febrero de 2017

Aceptado: 22 de agosto de 2018 